\title{
GIACINTO MORERA
}

Il giorno 8 febbraio di quest' anno si spegneva in Torino, dopo bu eve malattia, it Prof. Giacinto Morera, ancora nel fiore della virilitá e della sua attività scientifica. Otto giorni prima aveva tenuto la sua ultima lezione di meccanica al Politecnico. Scompare con lui una delle intelligenze più acute e penetranti che abbiano illustrato in Italia nell' ultimo ventennio le scienze matematiche, scompare una bella figura di uomo retto e coscienzioso, di insegnante efficace.

Giacinto Morera nacque in Novara il 18 Lnglio 1856 da famiglia di agiati commercianti. Si laureó in Torino in ingegneria ed in matematiche pure, seguendo i corsi del D' ovidio, del Genocchi, dello Siacci, che pù particolarmente egli considerava come suo maestro. Fece in seguito corsi di perfezionamento a Pavia col Beltrami ed il Casorati, a Pisa col Betti ed il Dini, a Lipsia col Mayer ed il Klein. Tornato in patria e giá noto per pregevoli lavori, dopo un anno vinceva, appena trentenne, il concorso di professore ordinario di Meccanica razionale nella Università di Genova. Quì rimase quattordici anni, insegnando anche la Fisica matematica e coprendo le cariche di Preside e di Rettore dell Università. Chiamato a Torino nel 1900 ad insegnare Meccanica razionale e superiore, passava nello scorso anno alla cattedra di Meccanica superiore per assumere l'incarico della Meccanica razionale al Politecnico. La facolta di scienze lo aveva da poco eletto suo Preside.

Fu membro dell' Accademia delle Scienze di Torino e Socio nazionale dell' Accadernia dei Lincei.

L' rpoca in cui Giacinto Morera compiè i suoi studi fu una delle piü fortunate per le scienze matematiche in Italia. 
I grandi maestri che averano iniziato il nostro risorgimento scientifico erano ancora nella loro piena vigoria ed egli potè seguire le loro scuole fiorenti a Torino, a Pavia, a Pisa acquistandosi una cultura larga e sicura che gli permise di iniziare subito con successo le proprie ricerche originali. Non si può dire di lui che fosse dotato di mente largamente inventiva; il suo ingegno ebbe in prevalenza tendenze analitiche e critiche, per cui più che a creare nuove teorie si dedicò a perfezionare e completare teorie già iniziate. Ma in questo lavoro egli profuse tesori di ingegno e di energia. Una quantità di quistioni egli chiarì, semplificò o perfezionò, portando quasi sempre il contributo di vedute ingegnose ed originali. Talchè la sua produzione scientifica può dirsi critica nel senso più largo e fecondo, cioè non dedicata allo studio di minuziosi particolari, ma alla penetrazione e soluzione delle quistioni più difficili e complicate.

Questa tendenza del suo ingegno si rivelò anche in un carattere esteriore di molte sue pubblicazioni, che egli presentò in forma di lavori brevi e concettosi; dei quali poi particolarmente si compiaceva, ed in conformità del suo carattere sincero, la sua compiacenza non si tratteneva dal manifestare esplicitamente.

Allievo dello Siacci, fu attratto anzitutto dai problemi fondamentali della dinamica. Erano da poco apparsi, nel decennio dal 1872 al 1882, i lavori classici di Mayer, Lie, Frobenius, Darboux sulla teoria delle equazioni ai differenziali totali ed il problema famoso di Pfaff, che apprivano nuove vie e mettevano in luce relazioni nuove fra una quantità di quistioni diverse. Questi studi erano in diretta relazione coi metodi celebri a cui Hamilton e Jacobi avevano ricondotto la integrazione delle equazioni della dinamica. Il Morera acquistata una sicura padronanza di quei multiformi lavori, potè subito dare splendide prove della sua abilità analitica in un primo gruppo di lavori pubblicati nel 1882-83 negli Atti della Accademia di Torino e nei Rendicondi dell' Istituto Lombardo. Particolarmente importante fra questi è l'applicazione del metodo di Pfaff alla integrazione di un sistema jacobiano di equazioni a derivate parziali. 
Sopra questo genere di quistioni ritornò poi quasi venti anni dopo, studiando specialmente il problema della trasformazione delle equazioni canoniche del moto, in base alla teoria delle trasformazioni di contatto del Lie.

Un altro argomento studiato dal Morera con singolare predilezione fu quello delle funzioni armoniche ellissoidiche, a cui fu condotto dal problema di trovare una espressione della gravitá alla superficie del geoide. Generalizzando alcune formole del Prof. Pizzetti, egli riusci ad esprimere in modo assai semplice con integrali definiti le funzioni armoniche re. golari nel campo interno ed esterno ad un ellissoide, mediante le quali si puó risolvere il problema di Dirichlet. Anche in questi ultimi tempi, ispirandosi specialmente ai metodi mirabili dei geometri inglesi, egli andava pensando a quistioni di questa specie, e lasciò tra i suoi scritti un lavoro, quasi ultimato, sul problema della deformazione elastica di un ellissoide.

Le quistioni che si riferiscono alle proprietà dell' integrale di Cauchy nella rappresentazione delle funzioni di variabile complessa, alle discontinuita delle derivate delle funzioni potenziali, alla formola di rappresentazione di Gauss, furono trattate dal Morera in un bel gruppo di lavori, che per la forza dell' analisi, unita ad una precisione e limpidità singolare di esposizione, sono da considerarsi fra i migliori. Egli diede con questi un bell' esempio del modo con cui possono essere trattate queste delicate quistioni, nulla trascurando della necessaria esattezza, senza mai sovracaricare la trattazione di procedimenti ingombranti.

Il Morera ebbe pure un senso squisito delle applicazioni dell' analisi ai problemi naturali; aiutato in ciò anche dai suoi studi giovanili d'ingegneria, che gli avevano procurato una estesa cultura tecnica.

Lungo sarebbe il passare in rivista tutta la serie dei suoi lavori in argomenti di questa specie. Egli si occupò del problema della corda vibrante analizzando il significato delle varie formole integrali. Portò il suo contributo al problema della dimostrazione della formola, con cui Kirchboff rappresentò matematicamente il principio di Huygens, quando que- 
sto problema fu discusso fra noi dal Beltrami e dal Volterra. Studiò una espressione generale delle formole fondamentali della termodinamica senza specializzazione delle variabili indipendenti. Trattó anche della scarica oscillante fra le due armature di un condensatore.

Della teoria dell' elasticità si occupò quando erano vive le discussioni intorno al significato delle celebri formole di Maxwell e trovó delle nuove espressioni generali delle tensioni che soddisfanno alle equazioni dell' equilibrio. A questa teoria fu pure dedicato l' ultimo suo lavoro, comunicato all' Accademia di Torino nel marzo 1907. In esso egli studiava alcuni stati di tensione in cui puó trovarsi un corpo, per effetto di centri di pressione, senza che su di esso agiscano forze esterne e ne indicava una possibile applicazione per la rappresentazione dello stato di tensione, in cui si trovano le cosidette lagrime bataviche.

Amico di lui da più di un ventennio, collega suo ultimamente nella Facoltà di Scienze di Torino, abituato a conversare quasi giornalmente con lui di quistioni scientifiche ed universitarie, bo potuto intimamente apprezzarne le doti squisite di intelletto e di carattere, la serenità nel giudicare uomini e cose, la gaia festositá dell'animo che mai non lo abbandonava. Ne sento ora più profondamente la perdita. E questa è certamente grande per le Università Italiane che in poco più di un biennio videro scomparire ben sei egregi cul. tori della meccanica teoretica, Siacci, Bardelli, Ruffini, Cesàro, Picciati, Morera.

Estremamente alieno da qualunque esteriorità e da qualunque occupazione che non fosse scientifica o scolastica, Giacinto Morera fu conosciuto da pochi all'infuori del circolo della famiglia e dei colleghi. Ma fra questi la sua memoria rimarrà indimenticata, come i suoi scritti rimaranno una delle pagine migliori della scienza moderna italiana.

C. Somigliana. 